\title{
A cost-effective analysis of drug-eluting balloon in highly recurrent stenosis on dialysis vascular access
}

\author{
Telmo Carvalho ${ }^{1}$, Dulce Carvalho ${ }^{1}$, Patrícia Marujo ${ }^{1}$, Maria David ${ }^{1}$, João Cruz $^{1}$, Pedro Maia ${ }^{2}$, Pedro Ponce ${ }^{1}$ \\ ${ }^{1}$ Lisboa Vascular Access Centre, NephroCare Portugal, Lisboa, Portugal \\ ${ }^{2}$ Coimbra Vascular Access Centre, NephroCare Portugal, Coimbra, Portugal
}

\section{ABSTRACT}

Background: The efficacy and cost-effectiveness of drug-eluting balloon (DEB) in vascular access for dialysis remain unclear. Methods: Data obtained from using DEB in highly recurrent stenosis were retrospectively analyzed. These data were obtained from procedures performed in the Vascular Access Center, responsible for attending over 3600 patients from 30 dialysis centers. Highly recurrent stenosis is defined as stenosis having three or more previous interventions and/or associated with early recurrence defined as the last procedure in less than 90 days. Patency and cost-effectiveness were evaluated. Patency was defined as the number of days free of interventions after applying DEB. Cost-effectiveness was evaluated using the incremental cost-effectiveness ratio (ICER) and an estimation of costs per day/patient/year. Results: Data from 37 consecutive procedures on 32 patients were assessed. The average patency time between interventions was $107.22 \pm 104.428$ days before DEB intervention. After the procedure, the average patency time intervention was $160.27 \pm 96.472$ days $(\rho=0.001)$. The cost analysis revealed an ICER of 10.37€/ patency day gained. The costs estimation revealed improved patency of 14.5 days/patient/year, and an increased cost of 1028.4€/patient/year when compared to conventional balloon. Conclusions: This retrospective analysis may suggest a benefit of DEB on this highly-recurrent stenosis. Although it appears to be more expensive, the increased patency at long term may be considered encouraging.

Keywords: Drug-eluting balloon, Restenosis, Vascular Access.

\section{BACKGROUND}

Vascular access (VA) thrombosis is complex and usually associated to a variable degree of stenosis. Only $30 \%$ of stenosis involving $>50 \%$ vessel area may result in thrombosis ${ }^{1}$. Percutaneous transluminal angioplasty (PTA) may promote hyperplasia, resulting in restenosis ${ }^{1,2}$. In $20 \%$ of grafts, the restenosis occurs after one week and in $40 \%$ after one month ${ }^{3}$. The drug-eluting balloon (DEB) delivers an antiproliferative drug -paclitaxel $\left(+/-2 \mu \mathrm{g} / \mathrm{mm}^{2}\right)$, in single short inflation. The primary objective of using DEB is to delay or inhibit the development of hyperplasia which will lead to restenosis.

Data on the efficacy of DEB in VA have been growing in the last years, from small, retrospective, non-randomized to prospective multicenter studies ${ }^{4-6}$, demonstrating that angioplasty with DEB may contribute to improved patency of VA. DEB is more expensive than conventional balloons, however. From a decision-making viewpoint, this is relevant as all effective interventions regarding economic evaluation may not be accepted, as they could have an unacceptable cost.

Since 2008, Portugal has had a reimbursement system for hemodialysis characterized by a capitation system. In this system, the dialysis treatment providers accept a fixed payment for each patient per week, granting dialysis-related services, such as VA management ${ }^{7}$. The Vascular Access Center (VAC) Lumiar is responsible for attending over 3600 patients from 30 dialysis centres.
Since 2015, as DEB has been available, we have decided to apply these balloons to a particular type of stenosis, which is the stenosis that had been previously submitted to three or more interventions and/or associated to early recurrence defined as the last procedure having been performed within 90 days. We based this decision on two points. First, there are no definitive indications or guidelines on the use of DEB in VA stenosis. Second, the cost of DEB is three times higher than conventional balloon, considering the Portuguese market prices for these medical devices. After 13 months, we decided to evaluate whether this approach was cost-effective, particularly because we have selected highly recurrent stenosis.

This retrospective analysis aims to analyze the efficacy of DEB in this highly recurrent and previously treated stenosis, and the cost effectiveness of this approach.

\section{PATIENTS AND METHODS}

VAC has a registry of all VA from all patients performing dialysis, which includes type, location, date of creation, complications, and reinterventions (Vascular Access Registry (AV OnlineC). We conducted a retrospective and registry-based analysis of preexisting data from our database from all procedures performed in our VAC. All patients gave their prior consent to the procedure and for all data registry on the AV Online $@$ database. 
In a 13-month period, between the beginning of December 2015 to the end of December 2016, a total of 1147 PTAs were performed on hemodialysis outpatients.

The following data were collected: patient age and gender, the presence of diabetes, VA lifetime and location, type of vascular access, the location of stenosis, angiography referral criteria, number of previous percutaneous angioplasties (PTA), and number, type and date of re-interventions or VA failures after the procedure with DEB. Procedures with multiple stenoses and/or previous PTA with non-standard balloons (e.g. cutting and high pressure balloons) were excluded.

The referral criteria were: a reduction of intra-access flow (Qa) below $600 \mathrm{ml} / \mathrm{min}$ in arteriovenous graft (AVG) and below $400 \mathrm{ml} /$ min in arteriovenous fistula (AVF) and/or a reduction of Qa higher than $25 \%$ from the previous measurmente; increased intra-access pressures associated with decreased dialysis adequacy (Kt/V); ipsilateral VA-related edema; vena cava syndrome (SVCS); graft thrombosis and other motives (e.g. need for prolonged compression of the puncture site due to persistent bleeding, high venous pressure and difficult cannulation).

The Qa was measured using thermic dilution methodology with Blood Temperature Monitor (BTM ${ }^{\oplus}$ ), and $\mathrm{Kt} / \mathrm{V}$ was calculated by the Online Clearance Monitor $\left(\mathrm{OCM}^{\circledast}\right)$, both on Fresenius 5008 therapy system (Fresenius Medical Care AG, Bad Homburg, Germany). For the purpose of this analysis, the value of Qa considered before DEB intervention was the last Qa measured within the last month, and the Qa obtained up to one month after the DEB procedure. For Kt/V, we obtained the value from the dialysis session before the procedure with DEB and in the first treatment after DEB.

Stenosis was defined as significant if luminal diameter reduction $>50 \%$ vessel was visualized in the angiogram.

PTA was performed by a venous approach with Seldinger technique, using direct puncture of the vascular access, anterograde and/or retrograde with, using an 18 gauge (G) needle and placement of a 6 or 7 French percutaneous sheath introducer. No arterial puncture was performed in this study.

All these highly recurrent stenoses were treated by using Drug Coated Balloon PTA Catheter Lutonix Bard $035^{\circledR}$, which was used according to the leaflet recommendations. Briefly, the size of the DEB was decided based on diameter of the most proximal non-aneurysmal vessel. A pre-dilatation with a conventional balloon was performed to achieve $a \leq 30 \%$ residual stenosis. Then DEB was rapidly advanced to the target site in the shortest possible time (i.e. 30 seconds) and placed in order to cover the entire lesion proximally and distally beyond the pre-dilatation injury segment. It was inflated to appropriate pressure to ensure full wall apposition and the inflation maintained for a minimum of 2 minutes (120 seconds). No anticoagulant or other intravenous medications were used.

The final result was considered positive if reappearance of a continuous thrill over the access and disappearance of the stenosis and/ or reduction or fading of collateral circulation was achieved. At the end of the procedure, the flow was evaluated through the visual assessment of the disappearance of the contrast after its administration (rapid versus slow flow).

In VAC the size of Drug Coated Balloon PTA Catheter Lutonix Bard $035^{\circledR \otimes} 035$ available varied from $5 \mathrm{~mm}$ to $12 \mathrm{~mm}$ of diameter and from $40 \mathrm{~mm}$ to $80 \mathrm{~mm}$ length.

In cases of dilation-induced rupture, the dilation balloon would be again inflated locally at low pressure 6 to 8 atmosphere (atm) for periods of 10 minutes, up to 3 times. If the rupture persisted, stent placement was considered. Analgesia was provided by subcutaneous lidocaine administration at the site of stenosis, if possible.

Complications were categorized as major or minor in line with guidelines of the Society of Cardiovascular \& Interventional Radiology.

Two endpoints were established in this analysis. VA patency, defined as the number of days between a procedure with DEB and the next re-intervention. Cost-effectiveness analysis, assessed with the incremental cost-effectiveness ratio (ICER) expressed in Euros/days of patency ${ }^{8}$. In the absence of relevant cost-utility and quality of life information, ICER determined the direct extra costs of patency gain. Additionally, estimation of costs per day/patient/year for each type of procedure was performed. Direct health care expenditures considered only the costs of PTA with conventional balloon and with DEB. In the Portuguese market, the average cost of a DEB balloon is $900 €$, and conventional PTA balloon is $300 €$. The price of DEB already includes the single conventional balloon used for pre-dilation.

Data were analyzed with the IBM SPSS Statistics $20.0^{\complement}$, using the Wilcoxon signed-rank non-parametric test, Student's T parametric test and the Kaplan-Meier survival curve. The statistical analysis was performed comparing the data using DEB and the results from previous PTA procedures (the historical results) in the same group of patients.

\section{RESULTS}

In all the procedures, the patient had only one stenotic lesion. In AVF the lesion was in the cephalic arch or central vein. In AVG the stenosis was located in venous graft anastomosis or in the central vein.

A total of 37 procedures with DEB were performed (3.2\% of all PTA; Figure 1) on 32 patients, $62.5 \%$ had AVG. At the time of DEB application, the average vascular access lifetime was $1481.35 \pm 1036.08$ days, previously submitted to $5.89 \pm 3.81$ interventions, median 5.0 and a follow up after DEB $284.89 \pm 106.32$ days (Table I).

The stenotic distribution lesions were as follows: in the venous graft anastomosis (35.1\%; $n=14)$, cephalic arch $(27 \% ; n=10)$ and central veins (37.8\%; $n=13)$. The Qa decrease was the main reason for referral (43.2\%; n=16) (Table II).

After the initial intervention with DEB, 9.3\% patients $(n=3)$ had no additional interventions, $90.6 \%(n=29)$ had a new intervention (Figure 1). 


\section{Figure 1}

Summary of Intervention in 32 patients.

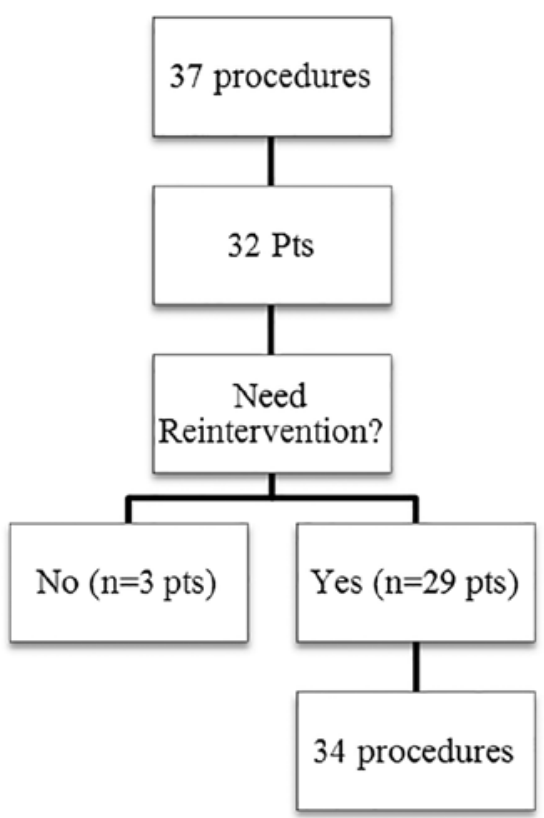

\section{$\underline{\text { Table I }}$}

Demographic data (Data are expressed as a percentage or mean $\pm \mathrm{sd}$ )

\begin{tabular}{|l|c|}
\hline Interventions & 37 \\
\hline Patients & 32 \\
\hline Female & $65.6 \%(n=21)$ \\
\hline Age & $68.4 \pm 16.42$ years \\
\hline AVF & $37.5 \%(n=12)$ \\
AVG & $62.5 \%(n=20)$ \\
\hline Diabetics & $62.5 \%(n=20)$ \\
\hline Time in dialysis (days) & $2802.31 \pm 2219.30$ days \\
\hline Age of vascular access (days) & $1481.35 \pm 1036.08$ \\
\hline Number of previous interventions & $5.89 \pm 3.81$ \\
\hline Mean of follow up after DEB (days) & $284.89 \pm 106.32$ \\
\hline
\end{tabular}

In these 29 patients, 5 additional angioplasties with DEB were performed: one patient was submitted to 3 interventions, and 2 patients underwent 2 procedures. A total of 34 re-interventions were realized in these 29 patients: 29 endovascular interventions ( 5 with DEB and remaining with conventional balloons) and 5 surgeries (Table III). At the end of the follow-up, only one VA (brachial-cephalic fistula) was not patent.

Before the procedure with $D E B$, the mean patency time between interventions was $107.22 \pm 104.428$ days. After DEB procedure, the average patency time significantly increased to $160.27 \pm 96.472$ days (Wilcoxon signed-rank test, $\rho=0.001$, Table IV). No statistical significance was found for mean patency according to location of stenosis,

\section{Table II}

Criteria referral

\begin{tabular}{l|c} 
Limb edema access (no surgery-related) & $18.92 \%(n=7)$ \\
\hline $\begin{array}{l}\text { Decrease in access flow rate } \\
\text { (<400ml/min AVF and < 600ml/min AVG) (Qa)* }\end{array}$ & $43.24 \%(n=16)$ \\
Increased intra-access pressures, with reduced efficiency dialysis & $10.81 \%(n=4)$ \\
\hline SVC syndrome & $5.41 \%(n=2)$ \\
\hline Graft thrombosis & $2.7 \%(n=1)$ \\
\hline Other motives & $18.92 \%(n=7)$
\end{tabular}

${ }^{*}$ Qa: the last measured Qa obtained up to one month before DEB intervention and one month after the procedure was considered.

\section{Table III}

Type of Reinterventions

\begin{tabular}{l||c} 
Surgery & \\
ePTFE bridge & $5.9 \%(n=2)$ \\
Vascular access surgical revision & $5.9 \%(n=2)$ \\
Vascular access ligation & $2.9 \%(n=1)$ \\
Endovascular & \\
PTA & $67.6 \%(n=23)$ \\
PTA with stent & $2.9 \%(n=1)$ \\
Thrombolysis & $14.7 \%(n=5)$
\end{tabular}

\section{Table IV}

Patency Days, Kt/N and Qa before and after DEB intervention (mean \pm sd)

\begin{tabular}{l|c|c} 
& Before DEB intervention & After DEB intervention \\
\hline Patency days & $107.22 \pm 104.428$ & $160.27 \pm 96.472^{\mathrm{a}}$ \\
$\mathrm{Kt} / \mathrm{V}^{\mathrm{a}}$ & $1.89 \pm 0.77$ & $2.11 \pm 0.34^{\mathrm{b}}$ \\
$\mathrm{Q}^{\mathrm{b}} \mathrm{b}$ & $804.81 \pm 551.71$ & $1010.35 \pm 415.55^{\mathrm{c}}$
\end{tabular}

The results considered statistically significant if $p<0.05 ; 1 p=0.001 ; 2 p=0.001,3 p=\rho=0,009$;

${ }^{a} \mathrm{Kt} / \mathrm{V}$ value: the value obtained in the last treatment before the procedure with $D E B$ and in the first treatment after $\mathrm{DEB} ;{ }^{\mathrm{b}}$ Qa value: the last measured Qa obtained up to one month before DEB intervention and one month after the procedure was considered.

although a consistent trend to increase was demonstrated after DEB application. Before DEB intervention, median patency for central veins stenosis was 68 days, for the cephalic arch stenosis 75.5 days and for venous graft anastomosis stenosis was 91 days. After DEB procedure, median patency increased; for central veins median 160 , for cephalic arch, median 119 and for venous graft anastomosis, median 165 days.

The values of Kt/V and Qa obtained before and after DEB procedure was also statistically different (Table IV). Of note, analyzing referral criteria, stenosis located to cephalic arch was associated with the highest Kt/V reduction and venous anastomosis stenosis had the highest Qa decrease (Table V).

The Kaplan-Meier (KM) curve revealed higher patency for VA after DEB treatment (Figure 2). KM curve of percentage of stenosis free from reintervention for VA before DEB revealed at 90 days, 180 days, 240 days, 360 days respectively $40.5 \%, 10.8 \%, 8.1 \%$ and $2.7 \%$ Table VI). After DEB, it was at 90 days, 180 days, 240 days and 360 days respectively 


\section{Table V}

Qa and Kt/V accordingly location of stenosis, before and after DEB intervention

\begin{tabular}{l|c|c|c}
\multicolumn{1}{c|}{} & \multicolumn{2}{c}{ Before DEB intervention } & \multicolumn{2}{c}{ After DEB intervention } \\
\cline { 2 - 4 } & $\mathrm{Kt} / \mathrm{V}^{\mathrm{a}}$ & $\mathrm{Qa}^{\mathrm{b}}$ & $\mathrm{Ka}^{\mathrm{b}} / \mathrm{V}^{\mathrm{a}}$ \\
\hline Venous graft Anastomosis & $1.80 \pm 0.43$ & $416.67 \pm 168.76$ & $2.08 \pm 0.25$ \\
Cephalic arch & $1.64 \pm 0.89$ & $981.43 \pm 675.24$ & $1.98 \pm 0.51$ \\
Central veins & $2.27 \pm 1.0$ & $1026.10 \pm 466.59$ & $2.25 \pm 0.30$ \\
\end{tabular}

${ }^{\mathrm{a}} \mathrm{Kt} / \mathrm{V}$ value: the value obtained in the last treatment before the procedure with DEB and in the first treatment after DEB; ${ }^{\mathrm{b}} \mathrm{Q}$ a value: the last measured Qa obtained up to one month before DEB intervention and one month after the procedure was considered.

\section{Table VI}

Stenosis free from reintervention after DEB compared to previous PTA with standard balloons

\begin{tabular}{c||c|c} 
& Standard balloons & AFTER BEB \\
\hline 90 days & $40.5 \%$ & $75.7 \%$ \\
\hline 180 days & $10.8 \%$ & $37.8 \%$ \\
\hline 240 days & $8.1 \%$ & $29.7 \%$ \\
360 days & $2.7 \%$ & $16.2 \%$
\end{tabular}

Analysis was performed comparing the data using DEB and the results from previous PTA procedures (the historical results) in the same group of patients.

\section{Figure 2}

Kaplan-Meier curve before and after the use of DEB

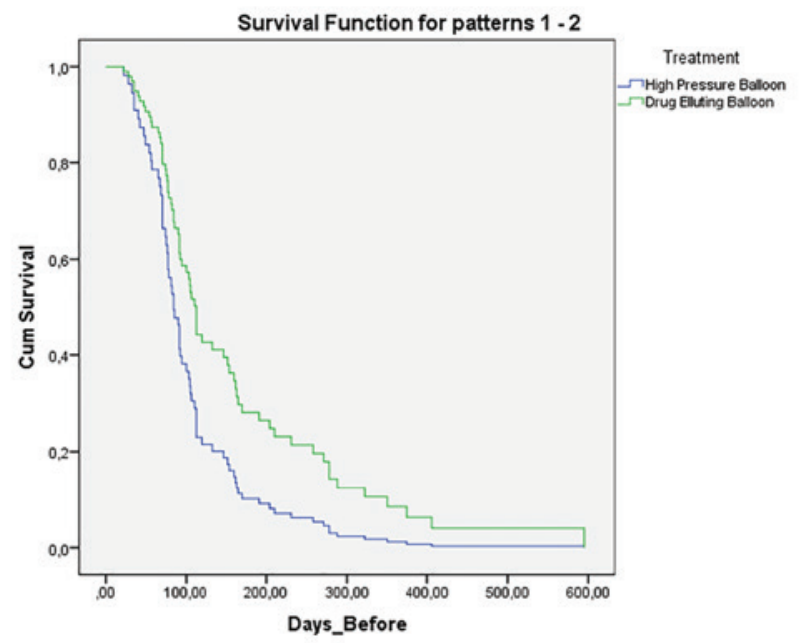

$75.7 \%, 37.8 \%, 29.7 \%$ and $16.2 \%$. Although this difference was not statistically different, the VA submitted to DEB procedure had a higher patency at all-time points (3 months to one year). Log rank (Mantel-Cox) revealed no statistical difference regarding the type of vascular access $(\rho=0.199)$ (Figure 3$)$ or the presence of diabetes $(\rho=0.303)$.

The analysis of cost revealed an ICER of $10.37 € /$ patency day gained with DEB procedure. Patency of VA was improved in 14.5 days/patient/

\section{Figure 3}

Kaplan-Meier curves after the use of DEB according to VA.

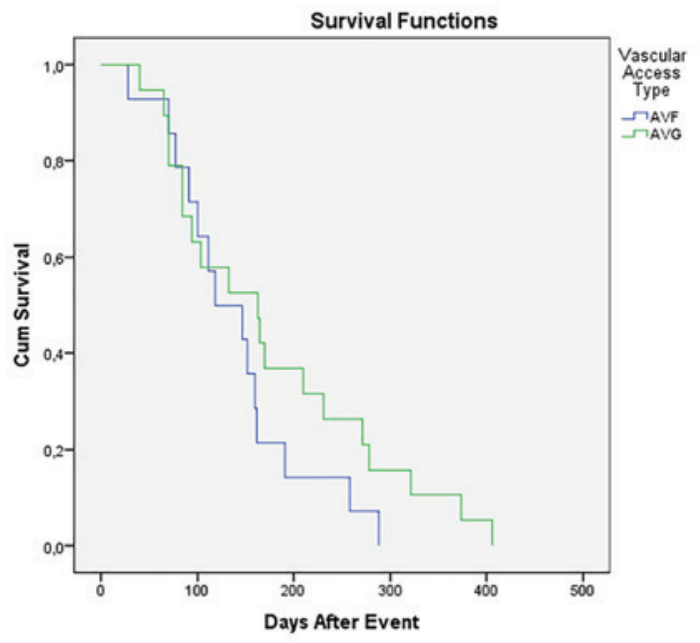

year with an additional fee of $1028.4 € /$ patient/year compared to conventional PTA balloon.

\section{DISCUSSION}

Vascular access dysfunction is recognized as an important cause of morbidity and mortality in hemodialysis patients, and consequently a burden for any health system. It is unclear whether an aggressive surveillance and monitoring of the vascular access program and preemptive intervention reduces thrombosis rates or eventually leads to an increased longevity of the $\mathrm{VA}^{9}$. Nevertheless, it may minimize the use of central venous catheters and reduce the vascular access-related hospitalizations. On the other hand, it is essential to preserve vascular area, as this is limited, conditioning the future of some patients and the need for chronic use of catheters.

Paclitaxel makes cells unable to proliferate and to move adequately and consequently may reduce the restenosis ${ }^{10}$. Available data from several studies have been showing that PTA with DEB may lead to longer patency and less re-intervention over uncoated balloon group $^{5,11-13}$. 
Of note, the resolution of stenosis, either recurrent or not, is left to the team's discretion, at VAC.This retrospective analysis intends to be a constructive and critical analysis of our approach.

Our study has analyzed 37 PTA with DEB on highly recurrent restenosis; the mean number of previous interventions with conventional balloons was 5.89 . The data showed that DEB significantly increased patency, Kt/V and Qa. However no statistical difference was found when data was examined according to the stenosis location. This may be explained by the small numbers in each group according to the location of stenosis. It is, however, noteworthy that a consistent increase in time free of intervention after DEB was found for all stenosis; for stenosis at venous and central venous anastomoses almost doubled, and in the cephalic arch, the patency was still higher but to a smaller extent. Stenosis located in the central vein and cephalic arch appears to be more challenging and the underlying stenosis in arch stenosis might be different from the others ${ }^{14}$.

$\mathrm{Kt} / \mathrm{V}$ and Qa were also compared. Stenosis located in the cephalic arch is associated with the highest reduction in Kt/V, not explained by the presence of collateral veins, as these were absent in these cases. We hypothesized that this stenosis might be either rapidly progressive or additionally affected by other factors.

Finally, our analysis was based on direct costs related to the different balloons and did not include all the costs, that is, the costs of losing the permanent access, the number of the collateral costs of lost dialysis days, hospitalization, number of days of work loss, the transportations to VAC and quality of life. It appears that DEB increases VA patency, incurring an additional cost of $1028.4 € /$ patient/year. Kitrou et al. have also considered the ICER, and demonstrated that the use of DEB was associated with 2198 euros per primary patency year of dialysis access gained $^{15}$. We wonder whether this will be additional cost, seeing as we have not considered all the costs. In addition, as DEB procedure was associated with an increased number of patency days, these may translate in lower costs at long-term follow up. In other words, in the short term, DEB appears to be more expensive, but at long-term may significantly reduce the number of procedures and consequently the costs.

We applied pre-dilation in all procedures as the leaflet recommends. Khawaja et al. showed that pre-dilation was not consistently considered in all studies or intervenions ${ }^{16}$. Drug Coated Balloon PTA Catheter Lutonix Bard was applied in all procedures in order to minimize bias between the procedures with DEB.

We recognise that our analysis has many limitations. It is a retrospective, non-randomized analysis, with a short follow up and includes a small number of procedures. Thus, some common evaluations, such as primary patency, secondary patency and assisted patency as defined by Society of Interventional Radiology (SIR) have not been performed. In addition, we have included AVF and AVG, as well as single stenosis from different locations, which might have different outcomes ${ }^{17}$. Furthermore, stenosis in central vein and arch cephalic seems to be more problematic and the length of stenosis has also been considered a predictor to access failure, but that it is not recorded in our databases ${ }^{18,19}$.

Interestingly, despite including VA previously submitted to multiple angioplasties, our data suggest a benefit to using DEB in these lesions.
We speculate whether the patency in lesions that have not been previously submitted to multiple interventions would lead to even better patency outcome.

Our approach also leads to increased direct costs but not an inconceivable additional cost to our VAC.

In spite of the increased amount of data showing the value of DEB for better patency of VA, its potential harms have been emergening. An increased risk of death at two and five years following the use of paclitaxel devices in the femoropopliteal artery of the lower limbs have been presented, which may raise problems and doubts of using it ${ }^{20}$. We wonder whether this is related to paclitaxel toxicity as multiple DEB are usually applied in peripheral arteries, as opposed to the reduced number of DEB applied to vascular access.

\section{Acknowledgments}

The authors thank the outstanding performance of all the surgeons and interventional nephrologists who contributed daily to these results.

Disclosure of potential conflicts of interest: none declared

\section{References}

1. Ponce $P$, Mateus A. Early Detection and Endovascular Intervention to Correct Dialysis Vascular Access Malfunction. Http://dx.doi.org/10.5772/intechopen.92631

2. Chi-Jen C, Po-Jen K, Lung-An H, Yu-Shien K, Yu-Lin K, Chin-Fen C et al. Highly increased cell proliferation activity in the restenotic hemodialysis vascular access after percutaneous transluminal angioplasty: implication in prevention of restenosis. Am J Kidney Dis. 2004; 43:74-84.

3. Moist LM, Churchill DN, House AA, Millward SF, Elliott JE, Kribs SW et al. Regular monitoring of access flow compared with monitoring of venous pressure fails to improve graft survival. J Am Soc Nephrol. 2003; 14:2645-2653.

4. Khawaja A, Cassidy DB, Shakarchi JA, McGrogan DG, Inston NG, Jones RG. Systematic review of drug eluting balloon angioplasty for arteriovenous haemodialysis access stenosis. J Vasc Access. 2016; 17:103 - 110 .

5. Swinnen JJ, Hitos K, Kairaitis L, et al. Multicentre, randomised, blinded, control trial of drug-eluting balloon vs Sham in recurrent native dialysis fistula stenoses. J Vasc Access. 2019; 20:260-269.

6. Lookstein RA, Haruguchi $\mathrm{H}$, Ouriel $\mathrm{K}$, et al. Drug-coated balloons for dysfunctional dialysis arteriovenous fistulas. N Engl J Med. 2020; 383:733-742.

7. Ponce P, Marcelli D, Guerreiro A, Grassmann A, Gonçalves C, Scatizzi L, et al. Converting to a capitation system for dialysis payment - the Portuguese experience. Blood Purif. 2012;34:313-324.

8. Stothers L. Cost-Effectiveness Analyses. In: Penson D.F., Wei J.T. (eds) Clinical Research Methods for Surgeons. Humana Press, 2006.

9. National Kidney Foundation. KDOQI Clinical Practice Guidelines and Clinical Practice Recommendations for 2006 Updates: Hemodialysis Adequacy, Peritoneal Dialysis Adequacy and Vascu- lar Access. Am J Kidney Dis. 2006; 48:S1-S322.

10. Portugaller RH, Kalmar PI, Deutschmann H. The eternal tale of dialysis access vessels and restenosis: are drug-eluting balloons the solution? J Vasc Access. 2014;15: 439-447.

11. Katsanos K, Karnabatidis D, Kitrou P, Spiliopoulos S, Christeas N, Siablis D. Paclitaxel-coated balloon angioplasty vs plain balloon dilation for the treatment of failing dialysis access: 6-month interim results from a prospective randomized controlled trial. J Endovasc Ther. 2012; 19:263-272.

12. Papachristou E, Siablis D, Karnabatidis D. Paclitaxel-coated versus plain balloon angioplasty for dysfunctional arteriovenous fistulae: one-year results of a prospective randomized controlled trial. J Vasc Interv Radiol. 2015; 26:348.

13. Liao MT, Chen MK, Hsieh MY et al. Drug-coated balloon versus conventional balloon angioplasty of hemodialysis arteriovenous fistula or graft: A systematic review and meta-analysis of randomized controlled trials. PLoS One 2020; https://doi.org/10.1371/journal.pone.0231463

14. Bennett S, Hammes MS, Blicharski T, Watson S, Funaki B. Characterization of the cephalic arch and location of stenosis. J Vasc Access. 2015; 16:13-18.

15. Kitrou PM, Katsanos K, Spiliopoulos S, Karnabatidis D, Siablis D. Drug-eluting versus plain balloon angioplasty for the treatment of failing dialysis access: final results and cost-effectiveness analysis from a prospective randomized controlled trial. Eur J Radiol. 2015;84: 418-423.

16. Khawaja AZ, Cassidy DB, Shakarchi JA, McGrogan DG, Inston NG, Jones RG. Systematic review of drug eluting balloon angioplasty for arteriovenous haemodialysis access stenosis. J Vasc Access. 2016; 17:103-110. 
17. AUYoung EW, Dykstra DM, Goodkin DA, Mapes DL, Wolfe RA. Hemodialysis vascular access preferences and outcomes in the Dialysis Outcomes and Practice Patterns Study (DOPPS). Kidney Int. 2002; 61:2266-2271.

18. Neuen BL, Gunnarsson R, Baer RA, Tosenovsky P, Green SJ , Golledge J, Mantha ML. Factors associated with patency following angioplasty of hemodialysis fistulae. J Vasc Interv Radiol. 2014; 25:1419-1426.

19. Heye S, Maleux G, Vaninbroukx J, Claes K, Kuypers D, Oyen R. Factors influencing technical success and outcome of percutaneous balloon angioplasty in de novo native hemodialysis arteriovenous fistulas. Eur J Radiol. 2012; 81:2298-2303.

20. Katsanos K, Spiliopoulos S, Kitrou P, Krokidis M, Karnabatidis D. Risk of death following application of paclitaxel-coated balloons and stents in the femoropopliteal artery of the leg: a systematic review and meta-analysis of randomized controlled trials. J Am Heart Assoc. 2018; 7:1-12.

\section{Correspondence to:}

Telmo Carvalho

Lisboa Vascular Access Centre, NephroCare Portugal, Lisboa, Portugal E-mail: telmo.carvalho@fmc-ag.com 